\title{
A phase II trial evaluating the efficacy and safety of perioperative pirfenidone for prevention of acute exacerbation of idiopathic pulmonary fibrosis in lung cancer patients undergoing pulmonary resection: West Japan Oncology Group 6711 L (PEOPLE Study)
}

Takekazu Iwata', Ichiro Yoshino ${ }^{1 *}$, Shigetoshi Yoshida' ${ }^{1}$, Norihiko Ikeda ${ }^{2}$, Masahiro Tsuboi ${ }^{3}$, Yuji Asato ${ }^{4}$, Nobuyuki Katakami ${ }^{5}$, Kazuhiro Sakamoto ${ }^{6}$, Yoshinori Yamashita ${ }^{7}$, Jiro Okami ${ }^{8}$, Tetsuya Mitsudomi ${ }^{9}$, Motohiro Yamashita ${ }^{10}$, Hiroshi Yokouchi ${ }^{11}$, Kenichi Okubo ${ }^{12}$, Morihito Okada ${ }^{13}$, Mitsuhiro Takenoyama ${ }^{14}$, Masayuki Chida ${ }^{15}$, Keisuke Tomii ${ }^{16}$, Motoki Matsuura ${ }^{17}$, Arata Azuma ${ }^{18}$, Tae Iwasawa $^{19}$, Kazuyoshi Kuwano ${ }^{20}$, Shuji Sakai ${ }^{21}$, Kenzo Hiroshima ${ }^{22}$, Junya Fukuoka ${ }^{23}$, Kenichi Yoshimura ${ }^{24}$, Hirohito Tada ${ }^{25}$, Kazuhiko Nakagawa ${ }^{26}$, Yoichi Nakanishi ${ }^{27}$ and West Japan Oncology Group

\begin{abstract}
Background: Idiopathic pulmonary fibrosis (IPF) often accompanies lung cancer, and life-threatening acute exacerbation (AE) of IPF (AE-IPF) is reported to occur in $20 \%$ of IPF patients who undergo lung cancer surgery. Pirfenidone is an anti-fibrotic agent known to reduce disease progression in IPF patients. A phase II study was conducted to evaluate whether perioperative pirfenidone treatment could reduce the incidence of postoperative AE-IPF patients with lung cancer.
\end{abstract}

Methods: Pirfenidone was orally administered to IPF patients who were candidates for lung cancer surgery; pirfenidone was dosed at $600 \mathrm{mg} /$ day for the first 2 weeks, followed by $1200 \mathrm{mg} /$ day. Surgery was performed after at least 2 weeks of 1200-mg/day administration. The primary endpoint was non-AE-IPF rate during postoperative days 0-30, compared to the null value of $80 \%$, and the secondary endpoint was safety. Radiologic and pathologic diagnoses of IPF and AE-IPF were confirmed by an independent review committee.

Results: From June 2012 to January 2014, 43 cases were enrolled, and 39 were eligible (full analysis set [FAS]). Both pirfenidone treatment and surgery were performed in 36 patients (per protocol set [PPS]). AE-IPF did not occur in 37/39 patients (94.9\% [95 \% confidential interval: $82.7-99.4 \%, p=0.01$ ]) in the FAS, and in 38/39 patients $(97.2 \%$ [95 \% confidential interval: 85.5-99.9\%, $p=0.004$ ] in the PPS. A grade 5 adverse event (death) occurred in 1 patient, after AE-IPF; no other grade 3-5 adverse events were observed.

(Continued on next page)

\footnotetext{
* Correspondence: iyoshino@faculty.chiba-u.jp

${ }^{1}$ Department of General Thoracic Surgery, Chiba University Graduate School

of Medicine, 1-8-1 Inohana, Chuo-ku, Chiba, Chiba, Japan

Full list of author information is available at the end of the article
} 
(Continued from previous page)

Conclusions: Perioperative pirfenidone treatment is safe, and is promising for reducing AE-IPF after lung cancer surgery in IPF patients.

Trial registration: This clinical trial was registered with the University Hospital Medical Information Network (UMIN) on April 16th, 2012 (Registration Number: UMIN000007774).

Keywords: Idiopathic pulmonary fibrosis, Acute exacerbation, Lung cancer, Surgery, Pirfenidone

\section{Background}

Idiopathic pulmonary fibrosis (IPF) is the most common interstitial lung disease, and has a histological appearance of usual interstitial pneumonia (UIP). IPF is known to be a risk factor for lung cancer [1] and is often observed in lung cancer patients. Life-threatening acute exacerbation (AE) of IPF (AE-IPF) may occur in association with cancer treatment, including radiotherapy, chemotherapy, and surgery, thereby severely restricting the therapeutic options for IPF-associated lung cancer. Among IPF patients who undergo surgery for lung cancer, postoperative AE-IPF is reported to occur in approximately $20 \%$, with an associated mortality of about $50 \%$ [2-5]. According to nationwide research conducted by the Japanese Association of Thoracic Surgery, AE-IPF is the leading cause of death within 30 days after lung cancer surgery in Japan [6]. Thus far, no reports of successful preventative methods for postoperative AE-IPF have been published. Several treatments, including intraoperative fluid balance control [7], postoperative ulinastatin [8], and preoperative methylpredonisolone and sivelestat [9], have been reported to have the potential to prevent postoperative AE; however, these were all evaluated in small, single-institute prospective or retrospective studies. A Japanese multi-institutional retrospective large cohort study revealed that none of the potential prophylactic treatments tested, including steroids, sivelestat, and ulinastatin, demonstrated an ability to prevent AE [10].

The Assessment of Pirfenidone to Confirm Efficacy and Safety in Idiopathic Pulmonary Fibrosis (ASCEND) study group recently reported that pirfenidone, oral anti-fibrotic agent, significantly reduced disease progression, as reflected by lung function, exercise tolerance, and progression-free survival, in a phase III trial in patients with IPF $[11,12]$. However, with respect to postoperative AE-IPF, the utility of pirfenidone has not yet been evaluated. Therefore, we conducted the phase II West Japan Oncology Group (WJOG) 6711 L study, called the "Perioperative pirfenidone for lung cancer with fibrosis evaluation: PEOPLE study," to evaluate the safety and efficacy of perioperative pirfenidone treatment in the reduction of postoperative AE-IPF in patients with lung cancer.

\section{Methods}

Eligibility criteria

Patients were required to have the UIP/possible UIP pattern criteria published by ATS/ERS/JRS/ALAT in 2011 [13] by high-resolution computed tomography (HR-CT). Other inclusion criteria were: age 20 to 75 years; cytological, histological, or radiological diagnosis of non-small cell lung cancer; tolerability of general anesthesia; cancer lesion(s) resectable by single lobectomy or lesser resections; predicted post-surgical forced expiratory volume in 1 second $\geq 1000 \mathrm{~mL}$; able to receive orally administered drugs; and Eastern Cooperative Oncology Group performance status 0-1. Patients were ineligible if they had a history of previous thoracotomy/video-assisted thoracoscopic surgery (although patients who had a history of surgical biopsy for diagnosing IPF $>6$ months previously were accepted); history of IPF treatment (including pirfenidone, steroids, erythromycin, $\mathrm{N}$-acetyl cysteine, neutrophil elastase inhibitors, and immunosuppressants); history of radiation therapy that included a lung in the treatment field or chemotherapy; other known causes of interstitial pneumonia (e.g., environmental exposure, connective tissue disease, and drug toxicity); severe comorbidities (active infectious disease, severe heart disease, uncontrolled diabetes mellitus, gastrointestinal bleeding, glaucoma, or psychiatric disease); history of drug allergy; or obvious history of acute exacerbation of IPF. Patients receiving oxygen therapy and pregnant or breastfeeding women were also excluded.

\section{Study design}

This was a multicenter, single-arm phase II study designed to evaluate the efficacy of perioperative pirfenidone treatment on the reduction of postoperative AE-IPF in Japanese patients with lung cancer. The primary endpoint was non-AE rate during postoperative days $0-30$, and the secondary endpoint was safety. The expected non-AE rate was set at $95 \%$, and the non-AEthreshold was set at $80 \%$, because the non-AE rate in patients who did not receive perioperative pirfenidone treatment was assumed to be $\leq 80 \%$ based on the literature $[2-5,14,15]$. A total of 42 patients were determined to reject the non-AE rate of $80 \%$ under the expectation of $95 \%$ with a power of $\geq 0.80$ and a one-sided alpha of 0.05 . 


\section{Central review}

Radiologic and pathologic diagnoses of IPF-AE were confirmed by a central review committee. To validate the institutional diagnosis of IPF, preoperative HR-CT and pathological samples obtained by surgery (non-cancerous lesions) were collected from each participating institute for central review. Two radiologists with expertise in interstitial lung disease (S.S. and T.I.) initially evaluated the HR-CT data independently and then developed a consensus report after discussion. Two pathologists with expertise in interstitial lung disease (J.F. and K.H.) initially evaluated the pathological samples independently and then developed a consensus report after discussion. These experts followed the international IPF diagnostic criteria published in 2011 [13]. The reports and discussions of the radiological/pathological diagnoses were reviewed and approved by all members of the central review committee, including the radiologists, pathologists, respiratory physicians with expertise in interstitial lung disease (A.A. and K.K.), and surgeons (S.Y. and T.I.). If the final diagnosis by HR-CT and pathological analysis was not definitively IPF, a multidisciplinary discussion was conducted among the radiologists, pathologists, respiratory physicians, and surgeons, and this group made a final diagnosis of IPF/not IPF.

Each participating institute diagnosed AE-IPF according to the definition published by the Japanese Respiratory Society [16] as follows: subjective worsening of dyspnea; new-onset bilateral ground-glass attenuation; significant worsening of partial pressure of oxygen in arterial blood; and exclusion of alternative causes of dyspnea and radiological changes, including pulmonary infection, pneumothorax, worsening of malignant tumor, pulmonary embolism, and heart failure. These criteria are very similar to the definition of the American Thoracic Society [17], although endobronchial aspiration and bronchoalveolar lavage were not required. Each incidence of postoperative AE-IPF declared by the researchers was also evaluated by the central review committee. Central review of HR-CT and clinical data were conducted through multidisciplinary discussion to determine whether or not the diagnosis of AE-IPF was appropriate.

\section{Drug administration}

Pirfenidone (Shionogi \& Co., Ltd., Osaka, Japan) at a dose of $600 \mathrm{mg} /$ day was orally administered to patients who planned to undergo surgery for the first 2 weeks; the dose was then increased to $1200 \mathrm{mg} /$ day for the next 2-4 weeks. The administration dose and dose-increase interval were established according to the protocol included in the Japanese pirfenidone package insert, which was based on the results of the Japanese preauthorizing phase III trial [18]. Surgery was performed after at least 2 weeks of administration of pirfenidone
$1200 \mathrm{mg} /$ day. Pirfenidone was given until the morning of the surgery day, and then re-started at $1200 \mathrm{mg} /$ day as soon as oral administration was permitted; in most cases, this was on the first postoperative day. The dose was increased to $1800 \mathrm{mg} /$ day if possible and was continued for as long as possible at least until the 30th postoperative day.

\section{Analysis}

In the primary analyses, the non-AE rate was based on the full-analysis set (FAS) following the intent-to-treat principle. $P$-values were calculated using binomial tests. A $p$-value $<0.05$ was considered significant. All statistical analysis was performed using SAS version 9.2 (SAS Institute, Cary, NC).

\section{Results}

\section{Patient characteristics}

From June 2012 to January 2014, 43 patients from 17 Japanese institutions were enrolled. Although all patients initiated pirfenidone therapy, 4 patients were deemed ineligible due to severe heart disease $(n=2)$, a history of chemotherapy $(n=1)$, and initiation of pirfenidone prior to registration $(n=1)$. Thus, 39 patients comprised the FAS. Patients included 33 males $(84.6 \%)$ with a median age of 68.0 (range, 52-75) years, and 38 patients had Hugh-Jones grade I/II activity (97.5\%). Among the FAS cases, two patients stopped pirfenidone administration before surgery due to patient request, and surgery was cancelled for one patient because metastatic lesions were identified on further examination. Therefore, both pirfenidone treatment and surgery were performed in 36 patients (per protocol set [PPS]). All of the PPS patients had received pirfenidone $1200 \mathrm{mg} /$ day for $>2$ weeks at the time of surgery. The mean time at which pirfenidone was stopped around surgery was $18.4 \mathrm{~h}$. Characteristics of FAS and PPS patients were very similar (Table 1 ). No patients had a history of gastroesophageal reflux disease. The surgical and pathological backgrounds are summarized in Table 2. Lobectomy was performed in 26 FAS patients (68.4\%) and 24 PPS patients (66.7\%). Segmentectomy was performed in seven patients (FAS, 18.4\%; PPS, $19.4 \%$ ), and wedge resection was performed in seven patients (FAS, $18.4 \%$; PPS, $19.4 \%$ ). Combined resections were performed in three patients (FAS, $7.9 \%$; PPS, $8.3 \%)$. Histologic types included squamous cell carcinoma (FAS, $n=18$ [47.4 \%]; PPS, $n=18$ [50 \%]), adenocarcinoma (FAS, $n=16$ [42.1\%]; PPS, $n=14$ [38.9\%]), and other minor types. Pathological cancer stages included stage I/II (FAS, $n=22 / 12 \quad[57.9 \% /$ $31.6 \%]$; PPS, 20/12 [55.6\%/33.3\%]). With respect to IPF diagnosis, the central review board diagnosed 39 of the 43 registered cases as IPF and the remaining 4 cases as not IPF (the latter were diagnosed as unclassifiable 
Table 1 Patient characteristics

\begin{tabular}{|c|c|c|}
\hline & FAS $(n=39)$ & PPS $(n=36)$ \\
\hline Gender, M/F & $33(84.6 \%) / 6$ (15.4 \%) & $30(83.3 \%) / 6(16.7 \%)$ \\
\hline Median age, years (range) & $68.0(52-75)$ & $68.0(52-74)$ \\
\hline Smoking history, ex/never & 38 (97.4 \%)/1 (2.6 \%) & 35 (97.2 \%)/1 (2.8 \%) \\
\hline Pack years & $61.09 \pm 32.07$ & $61.85 \pm 33.01$ \\
\hline Body mass index & $24.35 \pm 3.43$ & $24.41 \pm 3.57$ \\
\hline \multicolumn{3}{|l|}{ Activity } \\
\hline Hugh-Jones I & $29(74.4 \%)$ & $26(72.2 \%)$ \\
\hline Hugh-Jones II & $9(23.1 \%)$ & $9(25.0 \%)$ \\
\hline Hugh-Jones III & $1(2.6 \%)$ & $1(2.8 \%)$ \\
\hline Hugh-Jones IV & $0(0.0 \%)$ & $0(0.0 \%)$ \\
\hline Hugh-Jones V & $0(0.0 \%)$ & $0(0.0 \%)$ \\
\hline Fine crackle (audible) & 17 (43.6 \%) & $16(44.4 \%)$ \\
\hline Vital capacity (\%predicted) & $96.51 \pm 15.21$ & $97.25 \pm 15.25$ \\
\hline $\mathrm{FEV}_{1.0}$ (\% predicted) & $92.31 \pm 16.74$ & $92.81 \pm 17.24$ \\
\hline $\mathrm{DL}_{\mathrm{CO}}(\%$ predicted $)$ & $73.71 \pm 23.11$ & $73.88 \pm 24.45$ \\
\hline $\mathrm{KL}-6(\mathrm{U} / \mathrm{mL})$ & $1012.3 \pm 891.9$ & $1022.4 \pm 924.7$ \\
\hline
\end{tabular}

$D L_{C O}$ diffusing capacity of the lung for carbon monoxide, FAS full analysis set, $F E V_{1.0}$ forced expiratory volume in $1 \mathrm{~s}$, PPS per protocol set

interstitial pneumonia $[n=2]$, cellular nonspecific interstitial pneumonia $[n=1]$, and respiratory bronchiolitisassociated interstitial lung disease $[n=1])$. Compared to the central review by the professional radiologist/pathologist/respirologist, the accuracy of the preoperative radiological diagnosis of each institution was $90.7 \%$. Four not-IPF cases were included in the FAS and the PPS to adhere to the intent-to-treat principle. With respect to efficacy, we also selected the confirmed IPF cases from the PPS and analyzed them separately (PPS-IPF, $n=32$ ).

\section{Safety}

The safety of pirfenidone treatment in the preoperative phase was evaluated in all patients who received pirfenidone ( $n=43$, safety analysis set) (Table 3$)$. In the preoperative period, the following adverse events were observed: photosensitivity $(n=1)$, anorexia $(n=4)$, nausea $(n=4)$, somnolence $(n=1)$, dizziness $(n=2)$, malaise $(n=2)$, anemia $(n=2)$, hypoalubuminemia $(n=2)$, alkaline phosphatase elevation $(n=2)$, aspartate aminotransferase elevation $(n=3)$, alanine aminotransferase elevation $(n=3), \gamma$-glutamyltransferase (GGT) elevation $(n=5)$, creatinine elevation $(n=1)$, hyponatremia $(n=$ $3)$, and hyperkalemia $(n=1)$; however, all of these events were grade 1 or 2 according to the Common Terminology Criteria for Adverse Events (CTCAE) version 4.0 scale. Safety in the postoperative phase was evaluated in 37 patients in the FAS who underwent surgery. Fewer patients than those in the FAS who
Table 2 Patient surgical and pathological backgrounds

\begin{tabular}{|c|c|c|}
\hline Surgical procedure & FAS $(n=38)$ & $\operatorname{PPS}(n=36)$ \\
\hline Operation time (hours) & $3.36 \pm 1.23$ & $3.37 \pm 1.25$ \\
\hline Blood loss (g) & $138.6 \pm 126.2$ & $127.7 \pm 114.6$ \\
\hline \multicolumn{3}{|l|}{ Surgical procedure } \\
\hline Lobectomy & $26(68.4 \%)$ & $24(66.7 \%)$ \\
\hline Segmentectomy & 7 (18.4 \%) & 7 (19.4 \%) \\
\hline Wedge resection & 7 (18.4 \%) & $7(19.4 \%)$ \\
\hline Combined resection & $3(7.9 \%)$ & $3(8.3 \%)$ \\
\hline \multicolumn{3}{|l|}{ Residual tumor } \\
\hline RO & $36(94.7 \%)$ & $34(94.4 \%)$ \\
\hline R1 & $1(2.6 \%)$ & $1(2.8 \%)$ \\
\hline R2 & $1(2.6 \%)$ & $1(2.8 \%)$ \\
\hline Cancer pathology & FAS $(n=38)$ & $\operatorname{PPS}(n=36)$ \\
\hline \multicolumn{3}{|l|}{ Pathological type } \\
\hline Squamous cell carcinoma & $18(47.4 \%)$ & $18(50.0 \%)$ \\
\hline Adenocarcinoma & $16(42.1 \%)$ & $14(38.9 \%)$ \\
\hline Large cell carcinoma & $0(0.0 \%)$ & $0(0.0 \%)$ \\
\hline Adenosquamous carcinoma & $1(2.6 \%)$ & $1(2.8 \%)$ \\
\hline Other & $3(7.9 \%)$ & $3(8.3 \%)$ \\
\hline \multicolumn{3}{|l|}{ Pathological stage } \\
\hline IA & $10(26.3 \%)$ & $10(27.8 \%)$ \\
\hline $\mathrm{IB}$ & $12(31.6 \%)$ & $10(27.8 \%)$ \\
\hline$\| A$ & $8(21.1 \%)$ & $8(22.2 \%)$ \\
\hline$\| B$ & $4(10.5 \%)$ & $4(11.1 \%)$ \\
\hline$\| \mathrm{A}$ & $2(5.3 \%)$ & $2(5 . \%)$ \\
\hline$\| \mathrm{B}$ & $0(0.0 \%)$ & $0(0.0 \%)$ \\
\hline IV & $1(2.6 \%)$ & $1(2.8 \%)$ \\
\hline (non-cancer) & $1(2.6 \%)$ & $1(2.8 \%)$ \\
\hline
\end{tabular}

underwent surgery were evaluated for postoperative safety, because we did not require reporting of clinical data, except for the occurrence of AE-IPF, for patients who quit pirfenidone administration prior to surgery. The following adverse events were observed postoperatively: anorexia $(n=6)$, nausea $(n=3)$, diarrhea $(n=1)$, dizziness $(n=1)$, malaise $(n=2)$, respiratory failure $(n=1)$, dyspnea $(n=6)$, pulmonary fistula $(n=2)$, postoperative hemorrhage $(n=1)$, cough $(n=$ $1)$, atrial fibrillation $(n=1)$, wound dehiscence $(n=1)$, pneumonitis $(n=1)$, dysgeusia $(n=1)$, white blood cell decreased $(n=2)$, neutrophil count decreased $(n=1)$, anemia $(n=4)$, hypoalbuminemia $(n=19)$, alkaline phosphatase increased $(n=8)$, blood bilirubin increased $(n=1)$, aspartate aminotransferase increased $(n=8)$, alanine aminotransferase increased $(n=6)$, GGT increased $(n=6)$, hyponatremia $(n=8)$, hypokalemia $(n=3)$, and hypocalcemia $(n=3)$. Among these, 1 case of increased aspartate 
Table 3 Complications during the study

\begin{tabular}{|c|c|c|c|c|c|c|}
\hline \multirow[b]{3}{*}{ Photosensitivity } & \multicolumn{3}{|c|}{ Preoperative complications $(n=43)$} & \multicolumn{3}{|c|}{ Postoperative complications $(n=37)$} \\
\hline & \multirow{2}{*}{$\begin{array}{l}\text { Grade 1, } 2 \\
1\end{array}$} & \multicolumn{2}{|c|}{ Grade 3-5 } & \multirow{2}{*}{$\begin{array}{l}\text { Grade 1, } 2 \\
0\end{array}$} & \multicolumn{2}{|c|}{ Grade 3-5 } \\
\hline & & 0 & $0.0 \%$ & & 0 & $0.0 \%$ \\
\hline Anorexia & 4 & 0 & $0.0 \%$ & 5 & 1 & (Grade 4, $2.7 \%$ ) \\
\hline Nausea & 4 & 0 & $0.0 \%$ & 3 & 0 & $0.0 \%$ \\
\hline Diarrhea & 0 & 0 & $0.0 \%$ & 1 & 0 & $0.0 \%$ \\
\hline Somnolence & 1 & 0 & $0.0 \%$ & 0 & 0 & $0.0 \%$ \\
\hline Dizziness & 2 & 0 & $0.0 \%$ & 1 & 0 & $0.0 \%$ \\
\hline Malaise & 2 & 0 & $0.0 \%$ & 2 & 0 & $0.0 \%$ \\
\hline Respiratory failure & 0 & 0 & $0.0 \%$ & 0 & 1 & (Grade 4, $2.7 \%$ ) \\
\hline Dyspnea & 0 & 0 & $0.0 \%$ & 5 & 1 & (Grade 4, $2.7 \%$ ) \\
\hline Pulmonary fistula & 0 & 0 & $0.0 \%$ & 2 & 0 & $0.0 \%$ \\
\hline Postoperative hemorrhage & 0 & 0 & $0.0 \%$ & 1 & 0 & $0.0 \%$ \\
\hline Cough & 0 & 0 & $0.0 \%$ & 1 & 0 & $0.0 \%$ \\
\hline Atrial fibrillation & 0 & 0 & $0.0 \%$ & 1 & 0 & $0.0 \%$ \\
\hline Wound dehiscence & 0 & 0 & $0.0 \%$ & 1 & 0 & $0.0 \%$ \\
\hline Pneumonitis & 0 & 0 & $0.0 \%$ & 0 & 1 & (Grade 5, $2.7 \%$ ) \\
\hline Dysgeusia & 0 & 0 & $0.0 \%$ & 1 & 0 & $0.0 \%$ \\
\hline White blood cell decreased & 0 & 0 & $0.0 \%$ & 2 & 0 & $0.0 \%$ \\
\hline Neutrophil count decreased & 0 & 0 & $0.0 \%$ & 1 & 0 & $0.0 \%$ \\
\hline Anemia & 2 & 0 & $0.0 \%$ & 4 & 0 & $0.0 \%$ \\
\hline Hypoalbuminemia & 2 & 0 & $0.0 \%$ & 19 & 0 & $0.0 \%$ \\
\hline ALP increased & 2 & 0 & $0.0 \%$ & 8 & 0 & $0.0 \%$ \\
\hline Blood bilirubin increased & 0 & 0 & $0.0 \%$ & 1 & 0 & $0.0 \%$ \\
\hline AST increased & 3 & 0 & $0.0 \%$ & 8 & 1 & (Grade 3, $2.7 \%$ ) \\
\hline ALT increased & 3 & 0 & $0.0 \%$ & 6 & 0 & $0.0 \%$ \\
\hline GGT increased & 5 & 0 & $0.0 \%$ & 6 & 0 & $0.0 \%$ \\
\hline Creatinine increased & 1 & 0 & $0.0 \%$ & 0 & 0 & $0.0 \%$ \\
\hline Hyponatremia & 3 & 0 & $0.0 \%$ & 8 & 1 & (Grade 3, $2.7 \%$ ) \\
\hline Hyperkalemia & 1 & 0 & $0.0 \%$ & 0 & 0 & $0.0 \%$ \\
\hline Hypokalemia & 0 & 0 & $0.0 \%$ & 3 & 0 & $0.0 \%$ \\
\hline Hypocalcemia & 0 & 0 & $0.0 \%$ & 3 & 0 & $0.0 \%$ \\
\hline
\end{tabular}

ALP alkaline phosphatase, AST aspartate aminotransferase, $A L T$ alanine aminotransferase, GGT $\gamma$-glutamyltransferase

aminotransferase and 1 case of hyponatremia were reported as grade 3; 1 case of anorexia, 1 case of respiratory failure, and 1 case of dyspnea were reported as grade 4; and 1 case of pneumonitis was reported as grade 5 . The grade 4 respiratory failure and dyspnea and the grade 5 pneumonitis were determined to be due to AE-IPF.

Among the 43 registered patients who received pirfenidone, 41 cases underwent surgery (95.3\%). The reasons for the two surgery cancellations were discovery of metastatic lesions and necessity of cardiovascular surgery prior to lung surgery. Surgery was not cancelled in any patients due to pirfenidone-related adverse events.

\section{Efficacy}

AE-IPF was diagnosed in two patients (94.9\% [95 \% confidential interval (CI): 82.7-99.4 \%, $p=0.01$ under the null rate of $80 \%]$ ) in the FAS (Table 4). The central review board confirmed both of these cases as AE-IPF. Both patients died due to AE-IPF. One patient had quit pirfenidone administration prior to surgery; therefore, AE-IPF was recognized in only one patient (97.2\% [95 \% CI: 85.5-99.9\%, $p=0.004$ under the null rate of $80 \%]$ ) in the PPS. Among patients in the PPS, we also analyzed select IPF cases whose diagnoses were confirmed by the central review board (PPS-IPF). In the PPS-IPF, one 
Table 4 Efficacy

\begin{tabular}{llllll}
\hline Group & $N$ & AE-IPF occurred within the 30th POD & Non-AE rate within the 30th POD & $95 \%$ Cl & $p$-value \\
\hline FAS & 39 & 2 & $94.90 \%$ & $82.7-99.4$ & $p=0.010$ \\
PPS & 36 & 1 & $97.20 \%$ & $85.5-99.9$ & $p=0.004$ \\
PPS-IPF & 32 & 1 & $96.90 \%$ & $83.8-99.9$ & $p=0.008$ \\
\hline
\end{tabular}

$A E-I P F$ acute exacerbation of idiopathic pulmonary fibrosis, POD postoperative day, FAS full analysis set, PPS per protocol set, PPS-IPF PPS excluding non-IPF cases diagnosed by the central review committee

of the 32 patients experienced AE-IPF $(96.9 \%$ [95 \% CI: $83.8-99.9 \%, p=0.008$ under the null rate of $80 \%])$. When compared to the threshold derived from historical controls, AE-IPF occurred at a significantly lower incidence in this study.

\section{Discussion}

The current study was designed to evaluate the safety and efficacy of perioperative pirfenidone treatment in the reduction of postoperative AE-IPF in patients with lung cancer. No serious adverse events caused by pirfenidone were observed, and the incidence of AE-IPF among patients treated with pirfenidone was significantly lower than the null hypothesis.

The incidence of lung cancer have been reported to be $>7$-fold higher in IPF patients compared to the general population [1]. Conversely, IPF is detected in $3.7 \%$ to $8 \%$ of lung cancer patients [14, 19-21]. The prognosis of lung cancer with IPF is reported to be worse than that without IPF $[14,19,22]$. Moreover, lifethreatening AE-IPF sometimes occurs as a complication of lung cancer therapy. Radiation and chemotherapy are also known to be risk factors for AE-IPF [23-25]. Although the reason for the poor prognosis of lung cancer patients with IPF is not clear, therapeutic limitations associated with the potential of AE-IPF might at least partially account for the higher recurrence rate. If an effective strategy to reduce AE-IPF is established, more rational and radical treatment could be delivered to patients in a safer manner, which would lead to improved patient outcomes.

No studies have yet demonstrated that any agents can prevent or decrease the incidence of postoperative AE-IPF [20]. Pirfenidone is the first anti-fibrotic agent approved for the treatment of IPF [18]. The pooled data from the ASCEND and CAPACITY [26] trials showed that death related to IPF was significantly reduced among patients receiving pirfenidone therapy [12]. A phase II Japanese study found that pirfenidone prevented the development of AE-IPF [27]. However, these randomized studies evaluated the effect of pirfenidone in the context of the natural history of IPF. The effects of pirfenidone in patients undergoing anticancer therapy such as surgery, radiation, and chemotherapy, which are all risk factors for AE-IPF, have previously been unknown. We evaluated perioperative pirfenidone treatment within a single institute, and the results of this experience with a small sample size suggested that it is a feasible treatment and can be expected to reduce the incidence AE-IPF [28]. Therefore, we planned this multi-institutional prospective study to confirm our previous results.

Although AE-IPF is a well-known, life-threatening postoperative complication in patients with lung cancer accompanied by IPF, its reported incidence varies among studies; however, it generally ranges from $10 \%$ to $20 \%$ $[2-5,7,14,15,19,29,30]$. Compared to many small studies that evaluated 10-60 cases, Yoshimura et al. [5] reported the largest series of 205 IPF patients with lung cancer. In this study, these investigators observed that 41 patients $(20 \%)$ developed AE-IPF postoperatively, among which 29 (71 \%) died of respiratory failure due to AE-IPF. In the current study, an $80 \%$ non-exacerbation rate was set as the null hypothesis based on these previous reports, which were published prior to the planning of the current protocol in 2010; the non-AE rates in the current study of $94.9 \%$ (FAS), $97.2 \%$ (PPS), and $96.9 \%$ (PPS-IPF) were statistically positive compared to this null hypothesis.

Several studies have revealed that interstitial pneumonia subtype (UIP pattern or not) is a risk factor for postoperative AE-IPF [10, 31]. However, interstitial pneumonia subtypes were diagnosed with varying diagnostic criteria among different institutions in these previous studies. To circumvent this problem, we introduced a central review system to confirm the diagnosis of IPF following the international guidelines. As a result, we were able to show $\sim 90 \%$ diagnostic accuracy for each of the participating institutes. We believe that this first multi-institutional prospective study to evaluate postoperative AE-IPF after lung cancer surgery revealed reliable objective data about the incidence of AE-IPF; these data can be used as a standard when designing future studies.

\section{Limitations}

This study was not randomized but rather was a singlearm study in which historical data served as the control. When we combined the Japanese and international data for surgical lung cancer patients with idiopathic interstitial pneumonia published prior to the planning of the present study, the total incidence of AE was about $19 \%$ (data not shown); therefore, we believe the null hypothesis set herein was appropriate. However, patient background and 
the influence of some types of patient management that may have been better than those reported in the literature could not be controlled in such a single-arm study. Because the reported background risks for $\mathrm{AE}$ also varied among studies, it is difficult to verify the difference between the background risk in this study and those of previous studies. For example, respiratory function and activity shown by Hugh-Jones classification of the registered patients were relatively good in the current study. Severe IPF is undoubtedly considered to be a high risk; however, functionally inoperable cases were not included in this surgical study, and in many cases IPF was newly detected by surgeons during preoperative examination. Perhaps due to such a situation bias, the previously reported preoperative respiratory functions were also almost normal $[4,14,15,19,28-31]$. Postoperative AE occurs despite such surgical selection bias. To overcome such background problems and to confirm the efficacy of perioperative pirfenidone treatment for postoperative AE-IPF, a randomized study is required. Despite this limitation, we believe that the positive results of this phase II study justify the planning of a phase III randomized study.

\section{Conclusions}

In conclusion, this single-arm phase II study revealed that perioperative pirfenidone treatment is safe and promising for reducing AE-IPF after lung cancer surgery. These results encourage the planning of future confirmatory studies to compare pirfenidone to other treatments, such as nintedanib, for which the efficacy against progression of IPF has already been reported [32].

\section{Abbreviations}

AE: acute exacerbation; FAS: full analysis set; HR-CT: high-resolution computed tomography; IPF: idiopathic pulmonary fibrosis; JACS: Japanese Association of Chest Surgeons; PPS: per protocol set; UIP: usual interstitial pneumonia; WJOG: West Japan Oncology Group

\section{Acknowledgments}

The authors would like to thank Ms. Aya Yamanaka, Ms. Sachiko Nakayama, and Ms. Sachiko Akagi (all belong to WJOG Data Center, Osaka, Japan) for data management, and Dr. Shinichiro Nakamura (WJOG Data Center, Osaka, Japan) for oversight and management of the present study.

\footnotetext{
Authors' contributions

T.I. and Y.I. contributed to the study conception and design, acquisition and interpretation of data, drafting and critical review of the article, and approval of the final version for publication; N.I., M.T., Y.A., N.K., K.S., Y.Y, J.O., T.M, M.Y, H.Y., K.O., M.O., M.T., M.C., K.T., and M.M. contributed to the acquisition of data, critical review of the article, and approval of the final version for publication; S.Y., A.A., T.I., K.K., S.S., K.H., and J.F. contributed to the design and practice of central review, critical review of the article, and approval of the final version to be published; K.Y. contributed to the study conception and design, analysis and interpretation of data, drafting and critical review of the article, and approval of the final version for publication; and H.T., K.N., and Y.N. contributed to the study conception and design, critical review of the article, and approval of the final version for publication.
}

\section{Competing interests}

Y.I. received a study grant and A.A. received advisory board member fee from Shionogi Co. LTD for purposes outside of this study. All of the other authors declare that they have no competing interests.

\section{Ethics approval and consent to participate}

This study was conducted in accordance with the amended Declaration of Helsinki. The study protocol was approved by the WJOG Protocol Review Committee and Ethics Committee (Ref. ID WJOG6711L) and the institutional review board of each participating institution (Ref. IDs G24013: Chiba University Hospital, 2188: Tokyo Medical University Hospital, 2012-105: National Cancer Center Hospital East, 12-03: Institute of Biomedical Research and Innovation, 24-17: National Hospital Organization Kure Medical Center and Chugoku Cancer Center, 24-7: National Hospital Organization Yokohama Medical Center, 24-31: Ibaraki Prefectural Central Hospital, 1454: Fukushima Medical University Hospital, 45: Shikoku Cancer Center, 1208273056: Osaka Medical Center for Cancer and Cardiovascular Diseases, 24-157: Kinki University Hospital, 24-11: Hiroshima City Hospital, 1206-01: Kobe City Medical Center General Hospital, 24024: Dokkyo Medical University Hospital, 325: Hiroshima University Hospital, 2012-56: National Kyusyu Cancer Center, 24-36: Tokyo Medical and Dental University Hospital). This trial was registered with the University Hospital Medical Information Network (UMIN000007774). Written informed consent was obtained from all patients.

\section{Author details}

${ }^{1}$ Department of General Thoracic Surgery, Chiba University Graduate School of Medicine, 1-8-1 Inohana, Chuo-ku, Chiba, Chiba, Japan. ${ }^{2}$ First Department of Surgery, Tokyo Medical University Hospital, 6-7-1, Nishishinjuku,

Shinjuku-ku, Tokyo, Japan. ${ }^{3}$ Department of Thoracic Surgery, National Cancer Center Hospital East, 6-5-1, Kashiwanoha, Kashiwa, Chiba, Japan. ${ }^{4}$ Department of Thoracic Surgery, Ibaraki Prefectural Central Hospital, 6528, Kobuchi, Kasama, Ibaraki, Japan. ${ }^{5}$ Division of Integrated Oncology, Institute of Biomedical Research and Innovation, 2-2, Minatojima minamicho, Chuo-ku, Kobe, Hyogo, Japan. ${ }^{6}$ Department of Respiratory Surgery, National Hospital Organization Yokohama Medical Center, 3-60-2 Harajuku, Totsuka-ku, Yokohama, Kanagawa, Japan. ${ }^{7}$ Department of Respiratory Surgery, National Hospital Organization, Kure Medical Center and Chugoku Cancer Center, 3-1, Aoyamacho, Kure, Hiroshima, Japan. ${ }^{8}$ Department of General Thoracic Surgery, Osaka Medical Center for Cancer and Cardiovascular Diseases, 1-3-3 Nakamichi Higashinari, Osaka, Osaka, Japan. ${ }^{9}$ Division of Thoracic Surgery, Department of Surgery, Kinki University Faculty of Medicine, 377-2,

Ohnohigashi, Sayama, Osaka, Japan. ${ }^{10}$ Department of Thoracic Surgery, Shikoku Cancer Center, 160, Minamiumemotomachiko, Matsuyama, Ehime, Japan. ${ }^{11}$ Department of Pulmonary Medicine, Fukushima Medical University, 1, Hikarigaoka, Fukushima, Fukushima, Japan. ${ }^{12}$ Department of Thoracic Surgery, Tokyo Medical and Dental University, 1-5-45 Yushima, Bunkyo-ku, Tokyo, Japan. ${ }^{13}$ Department of Surgical Oncology, Hiroshima University, 1-2-3, Kasumi, Minami-ku, Hiroshima, Hiroshima, Japan. ${ }^{14}$ Department of Thoracic Oncology, National Kyushu Cancer Center, 3-1-1, Notame, Fukuoka Minami-ku, Fukuoka, Japan. ${ }^{15}$ Department of General Thoracic Surgery, Dokkyo Medical University, 880, Kitakobayashi, Shimotsugagun Mibumachi, Tochigi, Japan. ${ }^{16}$ Department of Respiratory Medicine, Kobe City Medical Center General Hospital, 2-1-1, Minatojimaminamimachi, Chuo-ku, Kobe, Hyogo, Japan. ${ }^{17}$ Department of Thoracic Surgery, Hiroshima City Hospital, 7-33, Motomachi, Naka-ku, Hiroshima, Hiroshima, Japan. ${ }^{18}$ Department of Pulmonary Medicine and Oncology, Graduate School of Medicine, Nippon Medical School, 1-1-5, Sendagi, Bunkyo-ku, Tokyo, Japan. ${ }^{19}$ Department of Radiology, Kanagawa Cardiovascular and Respiratory Center, 6-16-1, Tomiokahigashi, Kanazawa-ku, Yokohama, Kanagawa, Japan. ${ }^{20}$ Department of Internal Medicine, Division of Respiratory Medicine, Jikei University School of Medicine, 3-25-8, Nishi-Shinbashi, Minato-ku, Tokyo, Japan. ${ }^{21}$ Department of Diagnostic Imaging and Nuclear Medicine, Tokyo Women's Medical University, 8-1, Kawadacho, Shinjyuku-ku, Tokyo, Japan. ${ }^{22}$ Department of Pathology, Tokyo Women's Medical University Yachiyo Medical Center, 477-96, Ohwada-Shinden, Yachiyo, Chiba, Japan. ${ }^{23}$ Department of Pathology, Nagasaki University Graduate School of Biomedical Sciences, 1-12-4, Sakamoto, Nagasaki, Nagasaki, Japan. ${ }^{24}$ Innovative Clinical Research Center, Kanazawa University Hospital, 13-1, Takaramachi, Kanazawa, Ishikawa, Japan. ${ }^{25}$ Department of Thoracic Surgery, Suita Tokushukai Hospital, 21-1, Senriokanishi, Osaka, Japan. ${ }^{26}$ Department of Medical Oncology, Kinki University School of Medicine, 377-2, Ohnohigashi, Sayama, Osaka, Japan. 
${ }^{27}$ Research Institute for Diseases of the Chest, Graduate School of Medical Sciences, Kyushu University, 3-1-1, Maidashi, Higashi-ku, Fukuoka, Japan.

Received: 12 February 2016 Accepted: 6 July 2016

Published online: 22 July 2016

\section{References}

1. Hubbard R, Venn A, Lewis S, Britton J. Lung cancer and cryptogenic fibrosing alveolitis. A population-based cohort study. Am J Respir Crit Care Med. 2000;161:5-8.

2. Hyzy R, Huang S, Myers J, Flaherty K, Martinez F. Acute exacerbation of idiopathic pulmonary fibrosis. Chest. 2007;132:1652-8.

3. Hoshikawa Y, Kondo T. Perioperative lung injury: acute exacerbation of idiopathic pulmonary fibrosis and acute interstitial pneumonia after pulmonary resection. Nihon Geka Gakkai Zasshi. 2004;105:757-62.

4. Kumar P, Goldstraw P, Yamada K, Nicholson AG, Wells AU, Hansell DM, et al. Pulmonary fibrosis and lung cancer: risk and benefit analysis of pulmonary resection. J Thorac Cardiovasc Surg. 2003;125:1321-7.

5. Yoshimura K, Miyamoto A, Uruga H, Morokawa N, Beika Y, Kishi K. A nationwide survey on prophylaxis for acute exacerbation of idiopathic pulmonary fibrosis following lung surgery for associated lung cancer. The Annual Report by Study Group of Ministry of Health and Welfare for Diffuse Lung Disease. Diffuse Lung Diseases Research Group from the Ministry of Health, Labour and Welfare of Japanese Government 2008. 2009;77-81.

6. Masuda M, Kuwano H, Okumura M, Arai H, Endo S, Doki Y, et al. Thoracic and cardiovascular surgery in Japan during 2013. Gen Thorac Cardiovasc Surg. 2015;63:670-701

7. Mizuno Y, Iwata H, Shirahashi K, Takamochi K, Oh S, Suzuki K, et al. The importance of intraoperative fluid balance for the prevention of postoperative acute exacerbation of idiopathic pulmonary fibrosis after pulmonary resection for primary lung cancer. Eur J Cardiothorac Surg. 2012;41:e161-5.

8. Yamauchi Y, Izumi Y, Inoue M, Sugiura H, Goto T, Anraku M, et al. Safety of postoperative administration of human urinary trypsin inhibitor in lung cancer patients with idiopathic pulmonary fibrosis. PLoS One. 2011;6: e29053.

9. Ito H, Nakayama H, Yokose T, Yamada K. Prophylaxis for acute exacerbation of interstitial pneumonia after lung resection. Asian Cardiovasc Thorac Ann. 2014;8:948-54

10. Sato T, Teramukai S, Kondo H, Watanabe A, Ebina M, Kishi K, et al. Impact and predictors of acute exacerbation of interstitial lung diseases after pulmonary resection for lung cancer. J Thorac Cardiovasc Surg. 2014;147: 1604-11.

11. Iyer SN, Gurujeyalakshmi G, Giri SN. Effects of pirfenidone on transforming growth factor-beta gene expression at the transcriptional level in bleomycin hamster model of lung fibrosis. J Pharmacol Exp Ther. 1999;291:367-73.

12. King Jr TE, Bradford WZ, Castro-Bernardini S, Fagan EA, Glaspole I, Glassberg MK, et al. A phase 3 trial of pirfenidone in patients with idiopathic pulmonary fibrosis. N Engl J Med. 2014;370:2083-92.

13. Raghu G, Collard HR, Egan JJ, Martinez FJ, Behr J, Brown KK, et al. An official ATS/ERS/JRS/ALAT statement: idiopathic pulmonary fibrosis: evidence-based guidelines for diagnosis and management. Am J Respir Crit Care Med. 2011;183:788-824

14. Watanabe A, Higami T, Ohori S, Koyanagi T, Nakashima S, Mawatari T. Is lung cancer resection indicated in patients with idiopathic pulmonary fibrosis. J Thorac Cardiovasc Surg. 2008;136:1357-63.

15. Shintani Y, Ohta M, Iwasaki T, Ikeda N, Tomita E, Kawahara K, et al. Predictive factors for postoperative acute exacerbation of interstitial pneumonia combined with lung cancer. Gen Thorac Cardiovasc Surg. 2010;58:182-5.

16. Japanese Respiratory Society. Idiopathic interstitial pneumonias: diagnosis and treatment. 2nd ed. Tokyo: Nankodo; 2011. p. 67-73.

17. Collard HR, Moore BB, Flaherty KR, Brown KK, Kaner RJ, King Jr TE, et al. Acute exacerbations of idiopathic pulmonary fibrosis. Am J Respir Crit Care Med. 2007;176:636-43.

18. Taniguchi $H$, Ebina M, Kondoh Y, Ogura T, Azuma A, Suga M, et al. Pirfenidone in idiopathic pulmonary fibrosis. Eur Respir J. 2010;35:821-9.

19. Saito $Y$, Kawai $Y$, Takahashi N, Ikeya T, Murai K, Kawabata Y, et al. Survival after surgery for pathologic stage IA non-small cell lung cancer associated with idiopathic pulmonary fibrosis. Ann Thorac Surg. 2011:92:1812-7.

20. Watanabe A, Miyajima M, Mishina T, Nakazawa J, Harada R, Kawaharada N, et al. Surgical treatment for primary lung cancer combined with idiopathic pulmonary fibrosis. Gen Thorac Cardiovasc Surg. 2013;61:254-61.
21. Masuda M, Kuwano H, Okumura M, Amano J, Arai H, Endo S, et al. Thoracic and cardiovascular surgery in Japan during 2012: annual report by the Japanese Association for thoracic surgery. Gen Thorac Cardiovasc Surg. 2014;62:734-64.

22. Sato T, Watanabe A, Kondo H, Kanzaki M, Okubo K, Yokoi K, et al. Long-term results and predictors of survival after surgical resection of patients with lung cancer and interstitial lung diseases. J Thorac Cardiovasc Surg. 2015;149:64-9.

23. Watanabe N, Taniguchi H, Kondoh Y, Kimura T, Kataoka K, Nishiyama O, et al. Efficacy of chemotherapy for advanced non-small cell lung cancer with idiopathic pulmonary fibrosis. Respiration. 2013:85:326-31.

24. Minegishi $Y$, Sudoh J, Kuribayasi H, Mizutani H, Seike M, Azuma A, et al. The safety and efficacy of weekly paclitaxel in combination with carboplatin for advanced non-small cell lung cancer with idiopathic interstitial pneumonias. Lung Cancer. 2011:71:70-4.

25. Kenmotsu H, Naito T, Kimura M, Ono A, Shukuya T, Nakamura Y, et al. The risk of cytotoxic chemotherapy-related exacerbation of interstitial lung disease with lung cancer. J Thorac Oncol. 2011;6:1242-6.

26. Noble PW, Albera C, Bradford WZ, Costabel U, Glassberg MK, Kardatzke D, et al. Pirfenidone in patients with idiopathic pulmonary fibrosis (CAPACITY): two randomised trials. Lancet. 2011;377:1760-9.

27. Azuma A, Nukiwa T, Tsuboi E, Suga M, Abe S, Nakata K, et al. Double-blind, placebo-controlled trial of pirfenidone in patients with idiopathic pulmonary fibrosis. Am J Respir Crit Care Med. 2005:171:1040-7.

28. Iwata T, Yoshida S, Nagato K, Nakajima T, Suzuki H, Tagawa T, et al. Experience with perioperative pirfenidone for lung cancer surgery in patients with idiopathic pulmonary fibrosis. Surg Today. 2015;45:1263-70.

29. Sugiura H, Takeda A, Hoshi T, Kawabata Y, Sayama K, Jinzaki M, et al. Acute exacerbation of usual interstitial pneumonia after resection of lung cancer. Ann Thorac Surg. 2012;93:937-43.

30. Yano M, Sasaki H, Moriyama S, Hikosaka Y, Yokota K, Kobayashi S, et al. Postoperative acute exacerbation of pulmonary fibrosis in lung cancer patients undergoing lung resection. Interact Cardiovasc Thorac Surg. 2012;14:146-50.

31. Omori T, Tajiri M, Baba T, Ogura T, Iwasawa T, Okudela K, et al. Pulmonary resection for lung cancer in patients with idiopathic interstitial pneumonia. Ann Thorac Surg. 2015;100:954-60

32. Richeldi L, du Bois RM, Raghu G, Azuma A, Brown KK, Costabel U, et al. Efficacy and safety of nintedanib in idiopathic pulmonary fibrosis. N Engl J Med. 2014:370:2071-82.

\section{Submit your next manuscript to BioMed Central and we will help you at every step:}

- We accept pre-submission inquiries

- Our selector tool helps you to find the most relevant journal

- We provide round the clock customer support

- Convenient online submission

- Thorough peer review

- Inclusion in PubMed and all major indexing services

- Maximum visibility for your research

Submit your manuscript at www.biomedcentral.com/submit 